\title{
Auditory cortex activation is modulated by somatosensation in a case of tactile tinnitus
}

\author{
Kirsten Emmert • Dimitri Van De Ville • Philippe Bijlenga • \\ Dahila Amal Djema $\cdot$ Sven Haller
}

Received: 19 February 2014 / Accepted: 31 March 2014 / Published online: 13 April 2014

(C) Springer-Verlag Berlin Heidelberg 2014

Dear Sir,

We would like to draw your attention to some of our recent fMRI findings in a case of volitional tactile tinnitus modulation.

Tinnitus is a subjective auditory perception in the absence of external auditory stimuli. It affects millions of people worldwide [1]. The auditory percept of tinnitus can be continuous or pulsatile. Pulsatile tinnitus is commonly caused by vascular disease. In contrast, continuous tinnitus usually has no identifiable underlying vascular origin. The neural mechanisms underlying continuous tinnitus remain only partially understood, yet neuroimaging of tinnitus has often shown hyper-activation in some parts of the auditory region [2-4].

Many patients with tinnitus have individual strategies to modify the subjective tinnitus intensity, yet the neuronal mechanisms underlying this voluntary modification remains poorly investigated and objectified. Movements that can influence tinnitus include oral facial maneuvers (OFMs) [5], modulation of tinnitus by gazing in a certain direction (gazeevoked tinnitus, GET) [6], and more rarely tactile tinnitus (also known as cutaneous-evoked tinnitus, CET).

Most known cases of tactile tinnitus (three out of four cases) appeared after posterior fossa surgery [7-9]. Here, we report a thought-provoking case of a patient, who was

K. Emmert $(\bowtie) \cdot$ D. Van De Ville $\cdot$ D. A. Djema $\cdot$ S. Haller Department of Radiology and Medical Informatics, University of Geneva/Geneva University Hospital, Rue Gabrielle-Perret-Gentil, 4, 1211 Geneva 14, Switzerland

e-mail: kirsten.emmert@etu.unige.ch

K. Emmert • D. Van De Ville

Institute of Bioengineering, Ecole Polytechnique Fédérale de

Lausanne, Lausanne, Switzerland

P. Bijlenga

Department of Clinical Neurosciences, University of Geneva/Geneva University Hospital, Geneva, Switzerland examined with functional MRI, with tactile tinnitus without prior posterior fossa surgery in whom somatosensory stimulation of the right cheek increases the subjective tinnitus loudness on the right side, yet decreases loudness on the contralateral side.

The patient was a 55-year old right-handed female who reported a tinnitus that could be modified in intensity by tactile stimulation of the right cheek, specifically the dorsal part of the mandibular inferior to the right ear. Interestingly, the subject reported a decrease in tinnitus intensity in the left ear, while the subjective tinnitus on the right increased upon stimulation. At rest, the subject reported a left ear dominant, pulsatile tinnitus at a frequency of $8,000 \mathrm{~Hz}$. The subject's audiogram revealed near normal hearing thresholds. In addition, auditory-evoked potentials were normal as well as a standard electroencephalogram.

Images were acquired using a 3 Tesla Siemens Magnetom Trio Tim scanner (Erlangen, Germany) with a standard 32channel head-coil. A standard 3-D MP-RAGE sequence was acquired before functional imaging (auditory localizer and tactile task). In order to identify the individual primary auditory cortex region, a standard fMRI auditory block-design paradigm was performed (nine blocks, $20 \mathrm{~s}$ on/off) using standard echo-planar imaging $(\mathrm{EPI} ; \mathrm{TR}=2,500 \mathrm{~ms}, \mathrm{TE}=$ $30 \mathrm{~ms}$ flip angle $=90^{\circ}, 96$ by 96 matrix, 39 slices, $3-\mathrm{mm}^{3}$ isotropic voxels, interslice gap $0.3 \mathrm{~mm}, 152$ volumes for auditory localizer, 150 volumes for tactile task). Auditory stimulation consisted of a $1,000-\mathrm{Hz}$ sine tone pulsating at $6 \mathrm{~Hz}$ that is known to induce a strong blood oxygen leveldependent (BOLD) response in the auditory cortex.

Thereafter, two runs of tactile stimulation were performed in block-design using the same EPI sequence as the localizer. The seven blocks consisted of $25 \mathrm{~s}$ of rest (10 volumes, off) and $25 \mathrm{~s}$ of tactile stimulation (10 volumes, on) resulting in a run of about 6 min (375 s=150 volumes). During tactile stimulation periods, the subject touched her right cheek 
continuously with the right hand. During rest periods, the right arm rested close to the head without touching it.

The data was analyzed offline employing a general linear model (GLM) using BrainVoyager QX (Brain Innovation, Maastricht, The Netherlands) with conventional statistical analysis (type-I error control, false discovery rate at $p<0.05$ ) [10]. After identifying the region of interest (ROI) with the localizer, areas of activation and deactivation within or adjacent to the ROI were identified. Time course extraction and event-related averaging were performed for the identified areas.

As expected, the auditory stimulation during the localizer task evoked activation in the left and right auditory cortex (see Fig. 1a).

The tactile stimulation data showed an opposed modulation of activation within the left and right auditory cortex. While left ROI activation increased by about $1 \%$ (mean over stimulation period $1.02 \%$, standard error of the mean (SEM) $0.11 \%$ ), right ROI activation decreased by $1 \%$ of BOLD signal amplitude (mean $-1.05 \%$, SEM $0.13 \%$; see Fig. 2). The increased activation in the left ROI and the decreased activation in the right ROI correspond to the contralaterally perceived increase in tinnitus loudness to the right and decrease of loudness to the left ear reported by the subject.

In sum, we showed that the subjective perception of loudness change could be confirmed by an objective measurement -notably, fMRI BOLD responses within the auditory cortex. The perceived decreased tinnitus loudness on the left side and loudness increase on the right side due to right cheek tactile stimulation is accompanied by a corresponding change in activation in the contralateral auditory cortex. This seems plausible as auditory stimuli are predominantly processed on the contralateral side due to crossing fibers at the brainstem level.

Our study provides convincing evidence that extends previous findings in the literature. In particular, activity in the primary auditory cortex within association areas is modulated by tactile stimulation, showing a change in opposite directions for the left and the right side, while previous work [7] only showed a unilateral change. The involved regions are likely sites of somatosensory-auditory interaction due to multimodal input. As the first reported study was examining a case of strictly unilateral tinnitus including complete unilateral hearing loss due to posterior fossa surgery, results were likely to be biased by this pathology. In contrast, our study looked at a case of bilateral tinnitus with only mild hearing loss, which means that results are more likely to be transferable to normal tinnitus cases. As such, this tactile tinnitus case could suggest a possible model for changes in tinnitus loudness in general; i.e., tinnitus perception is modulated by contralateral auditory cortex activation.

Cross-modal plasticity is a common process, especially pronounced when deprivation of one sensory input system occurs in early ages as in a case of a congenitally deaf human adult who responded to vibrotactile stimulation with activation of somatosensory and auditory regions $[11,12]$, thus underlining the possible neuroplastic changes between somatosensory and auditory regions. Processes that were hypothesized to account for the increased cross-modal interactions are neuronal sprouting in reaction to neuronal damage (especially in cases with posterior fossa surgery) and unmasking of silent multimodal synapses.
Fig. 1 Regions of interest. a Analysis of the functional auditory localizer run resulted in detection of significant activation (orange) in the left and right auditory cortex. The activation clusters were used as ROI definitions for analysis of the tactile stimulation run. b ROIaveraged time course for the auditory localizer task

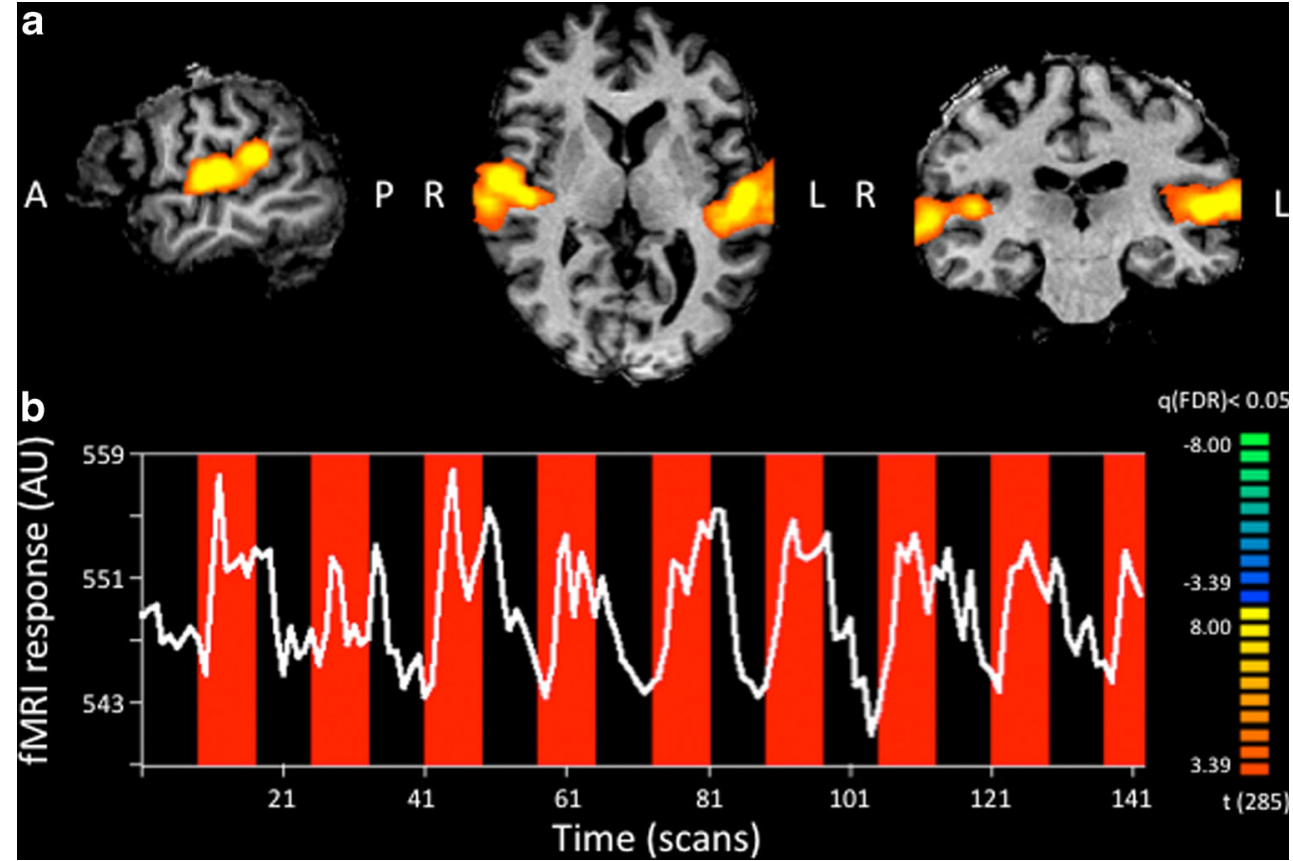



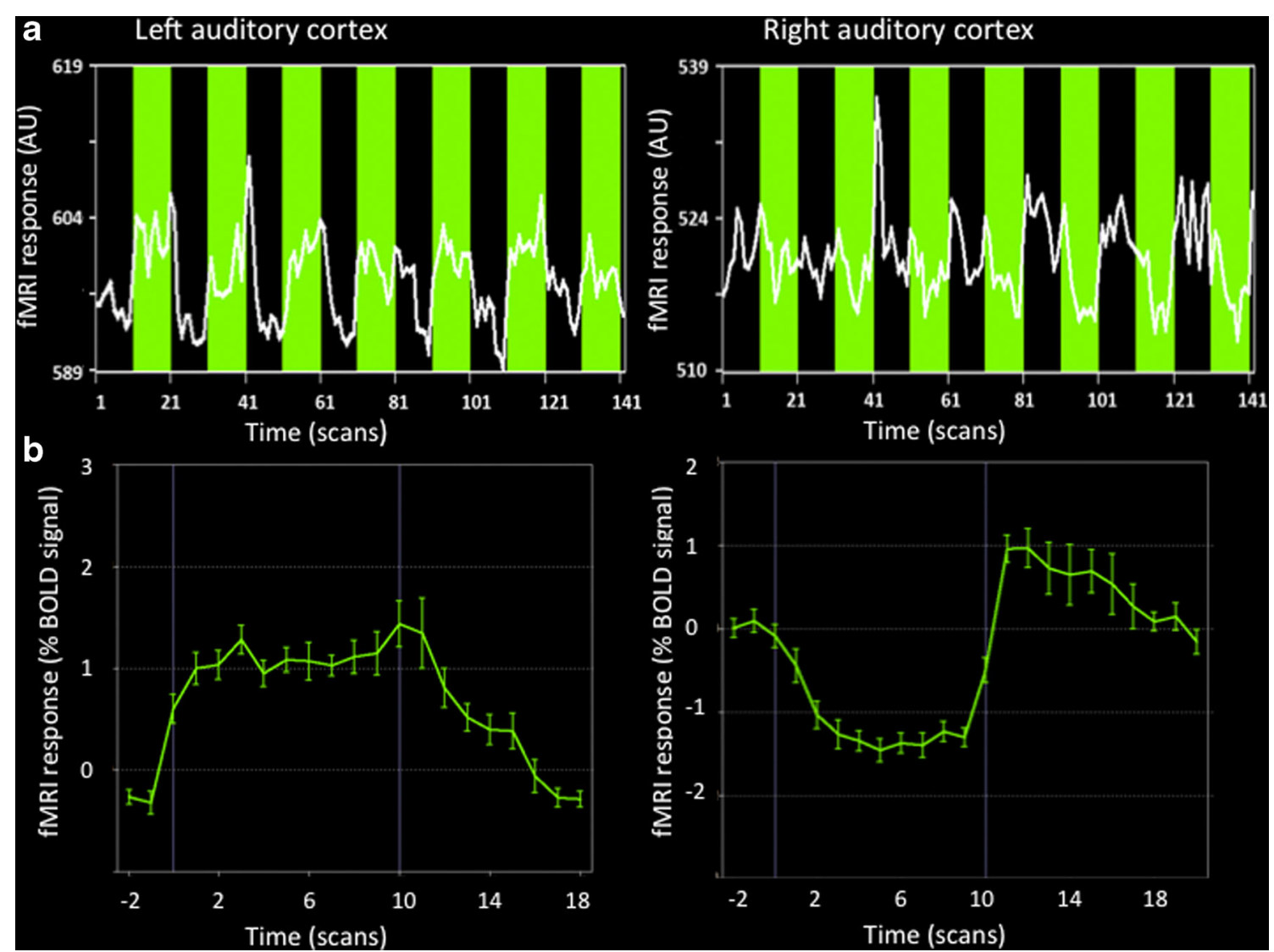

Fig. 2 Results for the tactile stimulation task. a Time courses of left and right auditory cortex activation (Talairach coordinates $\pm 57,-4,-1$ ) during right cheek tactile stimulation close to the identified primary cortex. A clear increase during tactile stimulation (green) can be seen for the left

Future research is needed to test how different brain areas are involved and interact in tinnitus modulation. More general studies of tinnitus show increased auditory cortex activation during tinnitus perception (e.g., [13]), which is in accordance with our results as increased loudness also led to an increase in activation in part of the auditory cortex. Due to the very small number of reported cases (5) and the even smaller size of individuals that underwent functional imaging (2), it is difficult to assess which mechanisms and areas could be involved in tactile tinnitus modulation. Moreover, detection power is low and activation differences in very restricted areas such as brainstem nuclei or the medial geniculate body could not be detected so far. In general, an involvement of the dorsal cochlear nucleus and extraleminiscal pathways seems possible for tactile tinnitus modulation while the somatosensory influence on the vestibular nucleus seems to be concentrated on eye movements $[14,15]$. As this case reported a tinnitus modulation caused by touching of the cheek, interactions of the trigeminal nerve with the auditory system would also be plausible.

Whether the modified auditory cortex activation is the origin of tactile tinnitus modulation or whether it is only mediating the effect remains to be determined. Due to the small number of patients with tactile tinnitus, a multi-centered,
ROI while there is a deactivation during stimulation for the right ROI which is also reflected in the average time course of all stimulation blocks. b Epoch-averaged BOLD responses where error bars indicate the SEM

large-scale search for other cases would be needed to set up a functional imaging study with a sufficient number of patients. To assess possible habituation effects of repeated tactile stimulation, as described for a GET case [16], a longer experiment with several fMRI sessions would be interesting. Functional imaging could be used to see if habituation is accompanied by a decrease in activation changes in the auditory network due to tinnitus modulation.

In conclusion, we found objective alterations of neuronal activation related to the subjective modification of tinnitus symptomatology in a case of tactile tinnitus. This observation might be useful when looking at other tactile tinnitus cases, as it might be possible to find objective correlates of their subjective tinnitus modulation as well as ultimately aiming at an objective diagnostic tool for tinnitus modulation. Importantly, the results of this study suggest that a decrease in auditory cortex activation is accompanied by a reduction in tinnitus emphasizing the importance of targeting the central auditory system activation in future tinnitus therapies. Due to the very small number of eported cases in particular implementing functional imaging, future research is needed to validate these findings and identify underlying mechanisms of somatosensory-auditory interactions. 
Ethical standards and patient consent We declare that all human and animal studies have been approved by the Commission cantonal d'éthique de la recherche and have therefore been performed in accordance with the ethical standards laid down in the 1964 Declaration of Helsinki and its later amendments. We declare that the patient gave informed consent prior to inclusion in this study.

Conflict of interest We declare that we have no conflict of interest.

\section{References}

1. Heller AJ (2003) Classification and epidemiology of tinnitus. Otolaryngol Clin North Am 2:239-248

2. Muhlnickel W, Elbert T, Taub E, Flor H (1998) Reorganization of auditory cortex in tinnitus. Proc Natl Acad Sci U S A 17:1034010343

3. Andersson G, Lyttkens L, Hirvela C, Furmark T, Tillfors M, Fredrikson M (2000) Regional cerebral blood flow during tinnitus: a PET case study with lidocaine and auditory stimulation. Acta Otolaryngol 8:967-972

4. Kleinjung T, Eichhammer P, Langguth B, Jacob P, Marienhagen J, Hajak G, Wolf SR, Strutz J (2005) Long-term effects of repetitive transcranial magnetic stimulation (rTMS) in patients with chronic tinnitus. Otolaryngol Head Neck Surg 4:566-569

5. Pinchoff RJ, Burkard RF, Salvi RJ, Coad ML, Lockwood AH (1998) Modulation of tinnitus by voluntary jaw movements. Am J Otol 19: 785-789
6. Wall M, Rosenberg M, Richardson D (1987) Gaze-evoked tinnitus. Neurology 37:1034-1037

7. Cacace AT, Cousins JP, Parnes SM, Semenoff D, Holmes T, McFarland DJ, Davenport C, Stegbauer K, Lovely TJ (1999) Cutaneous-evoked tinnitus. I. Phenomenology, psychophysics and functional imaging. Audiol Neurootol 4:247-257

8. Phillips JS, Baguley DM, Patel H, Moffat DA (2004) Tinnitus evoked by cutaneous stimulation. Neurology 63:1756

9. Bernstein JM (2007) Cutaneous-evoked tinnitus: first reported case without preceding posterior fossa surgery. Int Tinnitus J 13:159-160

10. Genovese CR, Lazar NA, Nichols T (2002) Thresholding of statistical maps in functional neuroimaging using the false discovery rate. Neuroimage 15:870-878

11. Levänen S, Jousmäki V, Hari R (1998) Vibration-induced auditorycortex activation in a congenitally deaf adult. Curr Biol 8:869-872

12. Levänen S, Hamdorf D (2001) Feeling vibrations: enhanced tactile sensitivity in congenitally deaf humans. Neurosci Lett 301:75-77

13. Langguth B, Eichhammer P, Kreutzer A, Maenner P, Marienhagen J, Kleinjung T, Sand P, Hajak G (2006) The impact of auditory cortex activity on characterizing and treating patients with chronic tinnitus -first results from a PET study. Acta Otolaryngol Suppl 556:84-88

14. Tomlinson RD, Robinson DA (1984) Signals in vestibular nucleus mediating vertical eye movements in the monkey. J Neurophysiol 51: $1121-1136$

15. Lockwood AH, Wack DS, Burkard RF, Coad ML, Reyes SA, Arnold SA, Salvi RJ (2001) The functional anatomy of gaze-evoked tinnitus and sustained lateral gaze. Neurology 56:472-480

16. Sanchez TG, Pio MRB (2007) The cure of a gaze-evoked tinnitus by repetition of gaze movements. Int Arch Otorhinolaryngol 11:345349 